\title{
Interferometric measurement of density in nonstationary shock wave reflection flow and comparison with CFD
}

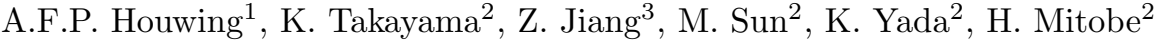 \\ 1 A.F.P. Houwing ALDiR Laboratory, Department of Physics, Faculty of Science, Australian National University, ACT 0200, \\ Australia (e-mail: Frank.Houwing@anu.edu.au) \\ 2 Shock Wave Research Center, Institute of Fluid Science, Tohoku University, Sendai, Aoba 980-8577, Japan \\ 3 Laboratory of High Temperature Gas Dynamics, Institute of Mechanics, Chinese Academy of Sciences, No. 15 Beisihuanxi \\ Road, Beijing, China (100080)
}

Received 12 November 2003 / Accepted 21 October 2004

Published online 31 March 2005 - (C) Springer-Verlag 2005

Communicated by R. Boyce

\begin{abstract}
We present density measurements from the application of interferometry and Fourier transform fringe analysis to the problem of nonstationary shock wave reflection over a semicircular cylinder and compare our experimental measurements to theoretical results from a CFD simulation of the same problem. The experimental results demonstrate our ability to resolve detailed structure in this complex shock wave reflection problem, allowing visualization of multiple shocks in the vicinity of the triple point, plus visualization of the shear layer and an associated vortical structure. Comparison between CFD and experiment show significant discrepancies with experiment producing a double Mach Reflection when CFD predicts a transitional Mach reflection.
\end{abstract}

Key words: Mach, reflection, interferometry, CFD

PACS: $47.40 .-\mathrm{x}, 42.40 . \mathrm{Kw}$

\section{Introduction}

As discussed by Caulfield [1], holography is a powerful flow diagnostic for investigating a variety of problems. Takayama [2] describes how holographic interferometry is used to study a variety of high speed flow problems of interest to physicists and engineers. The technique has been used extensively for both qualitative visualization and quantitative measurements of complex shock wave configurations. Both finite-fringe and infinite-fringe interferometry have been used. In many cases, the work has involved the latter type, which has been very useful for providing data for comparison with computational fluid dynamic (CFD) simulation. In the case of two-dimensional flows, comparison between CFD and experiment is reasonably straight-forward, since fringes in infinite-fringe interferograms correspond directly to density contours. However, spurious contributions to the phase shift can often cause problems when attempting to interpret such interferograms. In addition, their spatial resolution is limited by the fringe spacing, which can be quite large under certain flow conditions. To overcome these difficulties, finitefringe interferometry, accompanied by a good interferogram analysis technique, is arguably the best solution. In particular, for two-dimensional flows, it is possible to resolve complex flow structure with a high degree of spatial resolution, producing results suitable for detailed comparison with CFD simulations, provided one uses a sufficiently high heterodyning frequency during the interferometry and judiciously applies two-dimensional Fourier transform fringe analysis to provide phase maps and ultimately density maps of the flowfield. To demonstrate this, we investigate the nonstationary flow produced by the reflection of a planar shock wave at the upper surface of a semicircular cylinder as described below.

\section{Experimental setup}

The experimental setup used in our work consisted of a semicircular cylinder of diameter $D$, sitting on a pedestal of height $h$ as illustrated in Fig. 1 and mounted in the test section of a diaphragmless shock tube as described by Mitobe [3]. The test section containing the model was situated within the object beam of a double-exposure holographic interferometer illustrated in Fig. 2. In the flow visualization, the first exposure was made before the test time, and the second one was made during the time interval of interest to produce finite fringe holographic interferograms, which were later processed to produce the phase and density maps of the planar flowfield. 


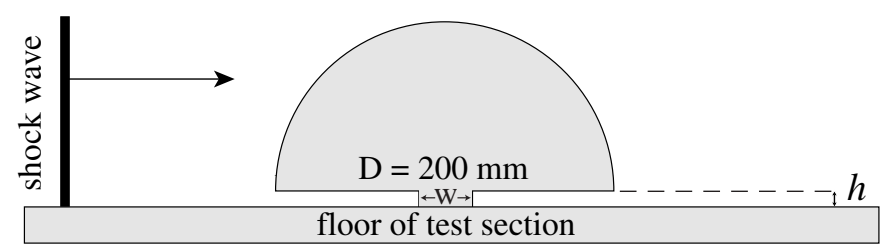

Fig. 1. Schematic of semicircular cylinder used in nonstationary shock reflection experiment. $h, w \approx 10 \mathrm{~mm}$

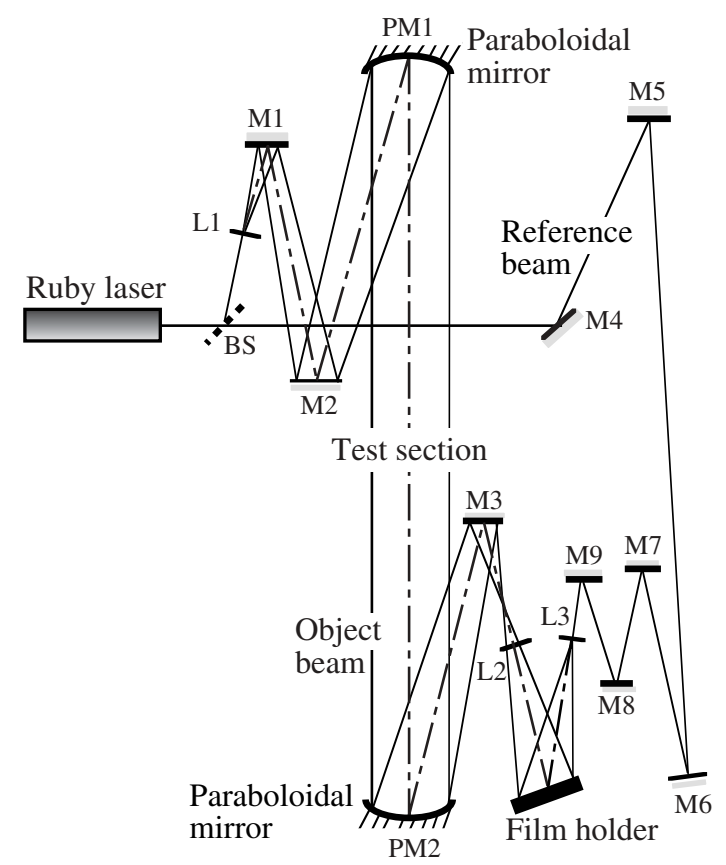

Fig. 2. Schematic of optical arrangement

The flow conditions for the experiments were for a shock wave of Mach number 2.33 travelling through pure molecular nitrogen gas at an initial pressure of $14.1 \mathrm{kPa}$, and an initial temperature of $292 \mathrm{~K}$. Flow conditions were such that perfect gas behavior with a ratio of specific heats of 1.4 could be assumed.

\section{Theoretical considerations}

The types of shock reflection that can be produced by interaction of an incident shock with a cylindrical surface are described by Ben-Dor et al. [4]. Two types are of particular interest to the current work: transitional Mach reflection (TMR) and Double Mach Reflection (DMR). These are illustrated in Figs. 3 and 4 respectively. As discussed by Ben-Dor et al. [5], TMR occurs when conditions are such that a compression wave downstream of the shock configuration can travel upstream and interact with the reflected shock to produce a kink $(\mathrm{K})$ that separates the reflected shock (r) from a second Mach stem $\left(\mathrm{m}^{\prime}\right)$. If the compression wave steepens into a shock wave, this kink develops into a second triple point $\left(\mathrm{T}^{\prime}\right)$ and DMR as illustrated in Fig. 4 is established.

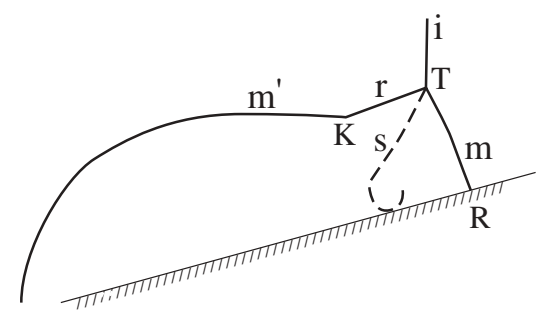

Fig. 3. Schematic of TMR, after Ben-Dor et al. [5]. i: incident shock; m: first Mach stem; r: reflected shock; $\mathrm{m}^{\prime}$ : second Mach stem; s: slipstream; R: reflection point; T: triple point; K: kink

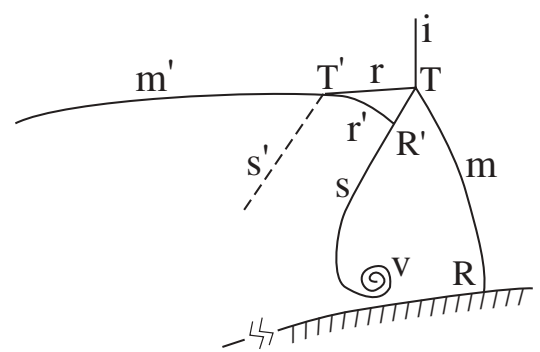

Fig. 4. Schematic of DMR, after Ben-Dor et al. [6]. i: incident shock; m: first Mach stem; r: first reflected hock; $\mathrm{m}^{\prime}$ : second Mach stem; $\mathrm{r}^{\prime}$ : second reflected shock; s: first slipstream; $\mathrm{s}^{\prime}$ : second slipstream. R: first reflection point; $\mathrm{R}^{\prime}$ : second reflection point; $\mathrm{T}$ : first triple point; $\mathrm{T}^{\prime}$ : second triple point; v: vortex

Table 1. Different cases simulated by CFD

\begin{tabular}{lllll}
\hline case & $p_{1}(\mathrm{kPa})$ & $M_{s}$ & $\frac{h}{D}$ & code \\
\hline $\mathrm{a}$ & 14.1 & 2.32 & 0 & $\mathrm{E}$ \\
$\mathrm{b}$ & 14.1 & 2.33 & 0 & $\mathrm{NS}$ \\
$\mathrm{c}$ & 14.1 & 2.35 & 0 & $\mathrm{E}$ \\
$\mathrm{d}$ & 14.1 & 2.33 & 0.05 & $\mathrm{NS}$ \\
$\mathrm{e}$ & 14.1 & 2.33 & 0 & $\mathrm{E}$ \\
\hline
\end{tabular}

\section{Numerical methods}

\subsection{CFD simulations}

Numerical simulations were performed for five different cases, with different values of initial pressure, $p_{1}$, incident shock Mach number $M_{s}$, normalized pedestal height $\frac{h}{D}$ and using different solvers, i.e., Euler (E) or Navier-Stokes (NS). These cases, which are identified in Table 1 are chosen to determine the sensitivity of the shock reflection to incident shock Mach number, viscous effects and the presence of the pedestal. By comparing the simulation results of cases a, c and e we can determine the sensitivity of the shock reflection to $M_{s}$, for an inviscid flow, for the range $2.32<M_{s}<2.35$. By comparing the simulation results of cases $\mathrm{b}$ and e, we are comparing results from a viscous simulation with those of an inviscid simulation, thereby determining the effects of viscosity on the reflection. By comparing simulation results from cases $\mathrm{b}$ and $\mathrm{d}$, we can determine the effect that the pedestal has on the shock reflection. 


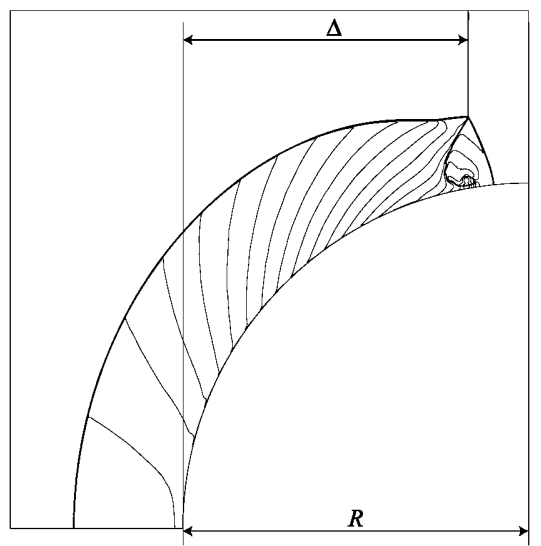

Fig. 5. Schematic illustrating how $\Delta$ and $R$ are defined

\subsection{Description of the CFD code}

The unsteady Euler equations are solved by the finite volume method on a solution-adaptive unstructured quadrilateral grid by an in-house algorithm described by Sun and Takayama [7], which has been successfully applied to a variety of gas dynamic problems.

In the present simulation, the two-step Runge-Kutta method is used to achieve second-order accuracy in time, and the second-order spatial accuracy is obtained by following the MUSCL-type extrapolation. Given two extrapolated states besides a cell interface, the numerical flux through it is given by a simple upstream splitting method described by Sun and Takayama [8] For the Navier-Stokes equations, the convection terms are discretized as done in the Euler equations, and other viscous and heat conductive terms are solved by the central difference scheme. The viscous flow simulation method is described in more detail by Sun et al. [9]. In this simulation, the coefficients of viscosity and heat-conduction varying with temperature are assumed to follow the Sutherland's formula.

\subsection{Computational grid}

The Initial background grid for the case of $\frac{h}{D}=0$ contains 147 cells covering the whole domain. The initial cell size is about $0.05 D$. The initial cells are automatically subdivided or refined around important flow features, such as shocks, vortices, and the refined cells can also be defined when these features pass away. An initial cell is allowed to be subdivided at most six times in the present computation, or a six level refinement is used. The finest cell after refining six times is about $\frac{0.05 D}{2^{6}}=0.00078 D$. To cover the domain with such a fine cell uniformly requires $147 \times 2^{6} \times 2^{6}=602,112$ cells. With the solutionadaptive technique, the simulation of shock reflection for cases where $\frac{h}{D}=0$ uses roughly 30,000 cells, only $6 \%$ of the uniform grid. The simulation for the case where $\frac{h}{D}=0.05$ is done with a similar number of cells and similar cell refinement.
Table 2. Type of reflection predicted by CFD as function of $\frac{\Delta}{R}$ for case b

\begin{tabular}{lllll}
\hline$\frac{\Delta}{R}$ & 0.207 & 0.413 & 0.612 & 0.825 \\
\hline type & RR & TMR & TMR & TMR
\end{tabular}

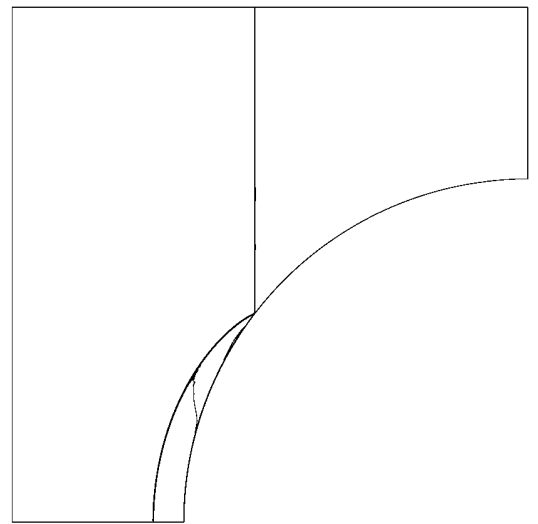

Fig. 6. Shock wave configuration at instant when $\frac{\Delta}{R}=0.207$ for case $b$

\section{Numerical simulations}

In this section, we present the results of our numerical simulations. We will present results for different instances in time as the shock configuration travels along the cylinder's surface. We have found that for the purpose of presenting our results, it is convenient to define these instances in terms of the ratio $\frac{\Delta}{R}$, where $\Delta$ is the distance moved in a horizontal direction by the incident shock after striking the stagnation point on the cylinder and $R$ is the radius of the cylinder. This convention is defined in Fig. 5.

\subsection{Sequence of simulation results for case b}

Figures 6, 7, 8 and 9 present density contours of the shock reflection as it travels along the surface of the cylinder for case b. In this numerical simulation, the shock configuration makes a transition from Regular Reflection (RR) to TMR when $0.207<\frac{\Delta}{R}<0.413$, with TMR persisting until $\frac{\Delta}{R}=0.825$. The results of this numerical simulation are summarized in Table 2.

\subsection{Simulations for different incident shock Mach numbers}

We performed CFD simulations for cases a, c and e to determine the sensitivity of the solution to the incident shock Mach number for inviscid flow. The resulting configuration at the instant when $\frac{\Delta}{R}=0.825$ for these cases is shown in Figs. 10, 11 and 13, respectively. The simulations for these three cases thus indicate that TMR is predicted when $\frac{\Delta}{R}=0.825$, with insensitivity to $M_{s}$ over the range $2.32<M_{s}<2.35$ 


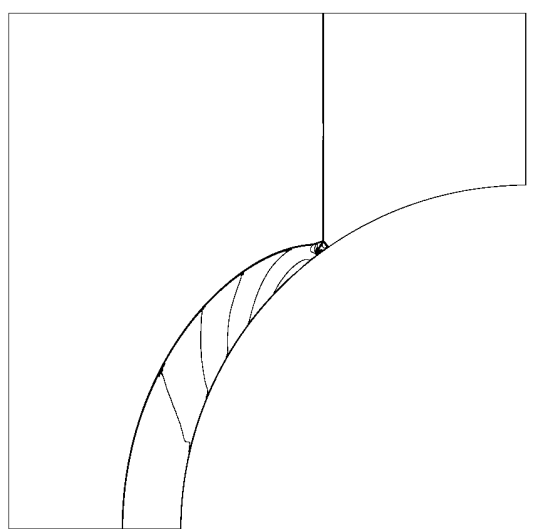

Fig. 7. Shock wave configuration at instant when $\frac{\Delta}{R}=0.413$ for case $b$

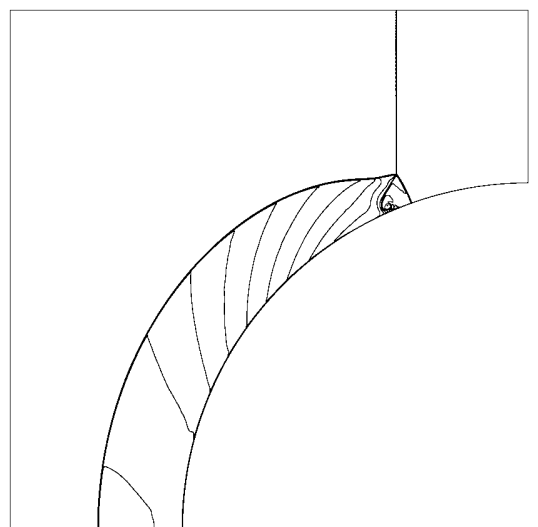

Fig. 8. Shock wave configuration at instant when $\frac{\Delta}{R}=0.612$ for case $b$

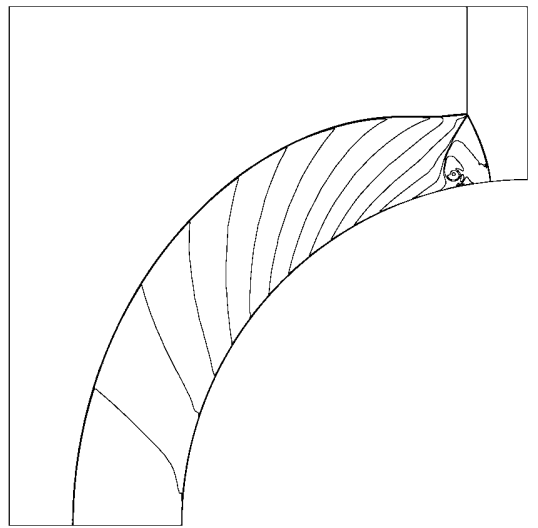

Fig. 9. Shock wave configuration at instant when $\frac{\Delta}{R}=0.825$ for case $b$

\subsection{Simulation for semicircular cylinder on the pedestal}

Case $d$ was simulated to determine the effects of the pedestal on the shock reflection configuration. We found that the numerical simulation for this case was very similar to that of case $\mathrm{b}$ if only the flows above the cylinder are compared. As an example, Fig. 12 is very similar to Fig. 9 above the cylinder, with both figures predicting a TMR configuration.

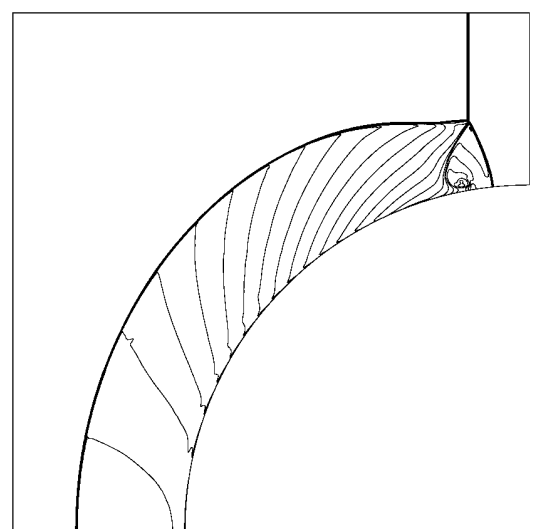

Fig. 10. Shock wave configuration at instant when $\frac{\Delta}{R}=0.825$ for case a

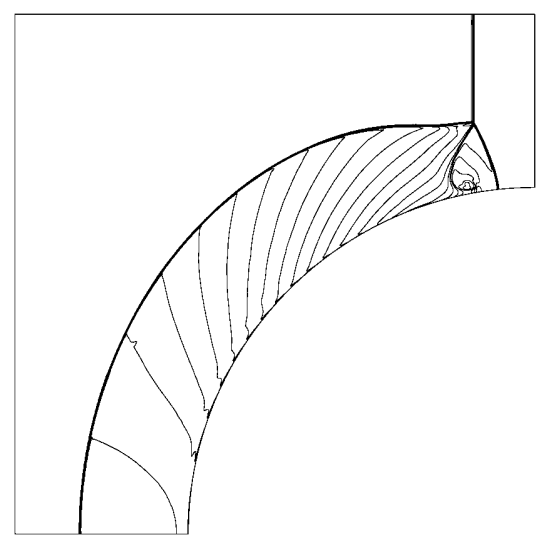

Fig. 11. Shock wave configuration at instant when $\frac{\Delta}{R}=0.825$ for case $\mathrm{c}$

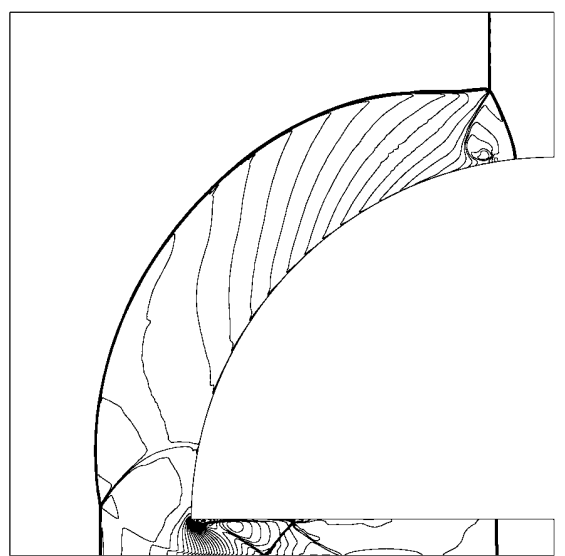

Fig. 12. Shock wave configuration at instant when $\frac{\Delta}{R}=0.825$ for case d

\subsection{Inviscid flow simulation}

Case e provides an inviscid flow simulation against which the viscous flow simulation of case $\mathrm{b}$ can be compared to ascertain whether there are viscous effects influencing what type of shock reflection is predicted by CFD. From our simulation, we found that cases $\mathrm{b}$ and e produced very similar results. In particular, when $\frac{\Delta}{R}=0.825$, simulations for both cases predicted TMR, as is evident by comparing Figs. 13 and 9. In summary, both viscous and inviscid flow 


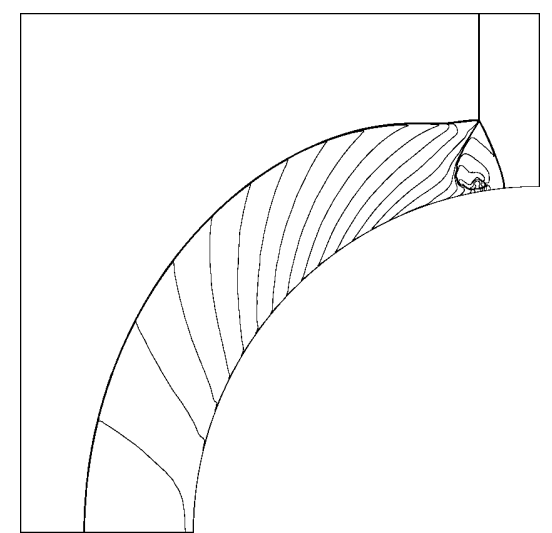

Fig. 13. Shock wave configuration for flow at instant when $\frac{\Delta}{R}=0.825$ for case $\mathrm{e}$

simulations predict TMR for the instant when $\frac{\Delta}{R}=0.825$, with no discernable sensitivity to shock Mach number and with the pedestal having no noticeable effect on the shock configuration.

\section{Sensitivity analysis}

We now make some remarks regarding the ability of our different simulations to explore the sensitivity of the reflection configuration at $\frac{\Delta}{R}=0.825$ to the initial conditions.

First, if an inviscid flow is assumed for the experiment, then the solution depends only on the shock Mach number. If the shock Mach number is fixed, the solution is determined, no matter what the value of the pressure. So, it is sufficient for us to show that the solution is not sensitive to the shock Mach number, which we have done.

Second, if viscosity and heat conductivity are influential in the process, it is sufficient to consider the NavierStokes equations. Then we should consider three parameters: Shock Mach number $M_{s}$; Reynolds number Re and Prandtl number $P r$. The last one, $P r$, varies with temperature. In the experiment, the characteristic temperature is just the room temperature that agrees with numerical simulation. Therefore, the Prandtl number can be neglected. The sensitivity of shock Mach number on the solution has been investigated in the inviscid simulation. The only one parameter left is the Reynolds number. We may argue that the inviscid solution corresponds to the case of infinite Reynolds number. Hence, the numerical simulations for cases a-e make up a complete and sufficient sensitivity analysis for our flow.

\section{Experimental method}

In this section, we provide further details about the experimental method used to obtain experimental results against which the CFD simulations are compared.

\subsection{Finite fringe holographic interferometry}

Here we present a brief description of the double-exposure finite-fringe holographic interferometry used in the current work. As shown in Fig. 2, a beam from a pulsed laser was split into two beams by a beam splitter (BS) to form the object beam and the reference beam. The object beam was collimated by a spherical lens (L1) and a paraboloidal mirror (PM1) before passing through the test section, after which the beam's diameter was reduced by the combination of another paraboloidal mirror (PM2) and another spherical lens (L2). The main purpose of PM2 and L2 was to image the flow region onto the holographic film. The film was secured on a flat film holder using a vacuum technique to ensure that the strict requirements of emulsion flatness were satisfied. The reference beam passed around the test section through reflection from a combination of mirrors (M4 to M9) before being expanded by a lens (L3) and illuminating the holographic film, where it interfered with the object beam.

With the type of interferometer described above, infinite fringe interferograms are produced by keeping the optics for both the reference and object beam fixed between exposures. In our work, finite-fringe interferograms were produced by tilting the reference beam through a small angle between exposures by precisely-controlled angular displacement of lens L3. As described above, the first exposure was made before the test event, and the second exposure was made during the test event, which corresponded to the instant when $\frac{\Delta}{R}=0.825$.

In the case of infinite fringe interferometry, the most significant phase shift that occurs is a result of the phase shift experienced by the object beam as it passes through the flow in the test section. In the case of the finite-fringe interferometry, the displacement of the reference beam produces an additional, linearly varying phase shift, that superimposes a heterodyning frequency over the whole image.

The holograms were recorded on Agfa AE75 film, with the pulsed laser source being a Ruby laser operating at a wavelength, $\lambda_{\exp }$, equal to $694.3 \mathrm{~nm}$. The holograms were reconstructed using a CW Argon-ion laser at a wavelength, $\lambda_{\text {rec }}$, of $514.5 \mathrm{~nm}$. The reconstructed interferograms were enlarged, printed, and then scanned with a high resolution scanner to produce digital images for later analysis.

\section{Overview of interferogram analysis}

The interferogram analysis technique used in the current work was based on the two-dimensional Fourier-transform method described by Bone et al. [10], Bone [11] and Babinsky and Takayama [12].

The method involved the following steps:

1) performing a two-dimensional Fourier transform of the interferogram;

2) applying a filter operation. This second step was required to remove both low-frequency and high-frequency noise from the data; 
3) performing a frequency shift in the Fourier transform plane, so that the data is located around zero frequency;

4) performing a two-dimensional inverse Fourier transform to produce the filtered inverse transform $g$;

5) determining the phase by evaluating the arctangent of the ratio of the imaginary and real parts of the inverse transform;

6) 'unwrapping' the phase by adding multiples of $2 \pi$ where appropriate. This step required a sophisticated search algorithm as described by Bone [11]; and

7) removing any residual background phase.

\section{Determining the density \\ from the unwrapped phase}

\subsection{Two-dimensional flows}

In the case of two dimensional flows, the phase $\phi$ is given by

$$
\phi(x, y)=\frac{2 \pi}{\lambda}\left[n_{\text {ref }}-n_{\text {flow }}(x, y)\right] W,
$$

where $W$ is the distance that the light travels through the phase-shifting medium, $\lambda$ is wavelength of this light, and $n$ is the refractive index. The subscripts 'ref' and 'flow' refer to 'reference' and 'flow' conditions, respectively. As discussed by Merzkirch [13], for a perfect gas of uniform composition, the refractive index can be related directly to the density, $\rho$, of the gas and its Gladstone-Dale coefficient $K$, so that one can write

$$
n=1+K \rho .
$$

Liepmann and Rohko [14] show that the Gladstone-Dale coefficient can be conveniently expressed in terms of its value at the standard density, $\rho_{s}$, in which case the above equation becomes:

$$
n=1+\beta \frac{\rho}{\rho_{s}} .
$$

Values for $\beta$ and $\rho_{s}$ for the gases used in the current work are given by Liepmann and Rohko [14]. Using the above equations, the density of the flow is determined to be given by

$$
\rho_{\text {flow }}-\rho_{\text {ref }}=\frac{\lambda \rho_{s}}{2 \pi W \beta}\left(\phi_{\text {ref }}-\phi_{\text {flow }}\right) .
$$

Figure 14 shows a $1024 \times 1024$ digital image of the finite-fringe interferogram of the planar flowfield investigated in the current work. The size of the imaged region is $94 \mathrm{~mm}$ in length $\times 134 \mathrm{~mm}$ in height.

\section{Analysis}

\subsection{Fourier transform}

This interferogram was processed using Steps 1-4 to produce the filtered phase distribution $g$. The Real part of this

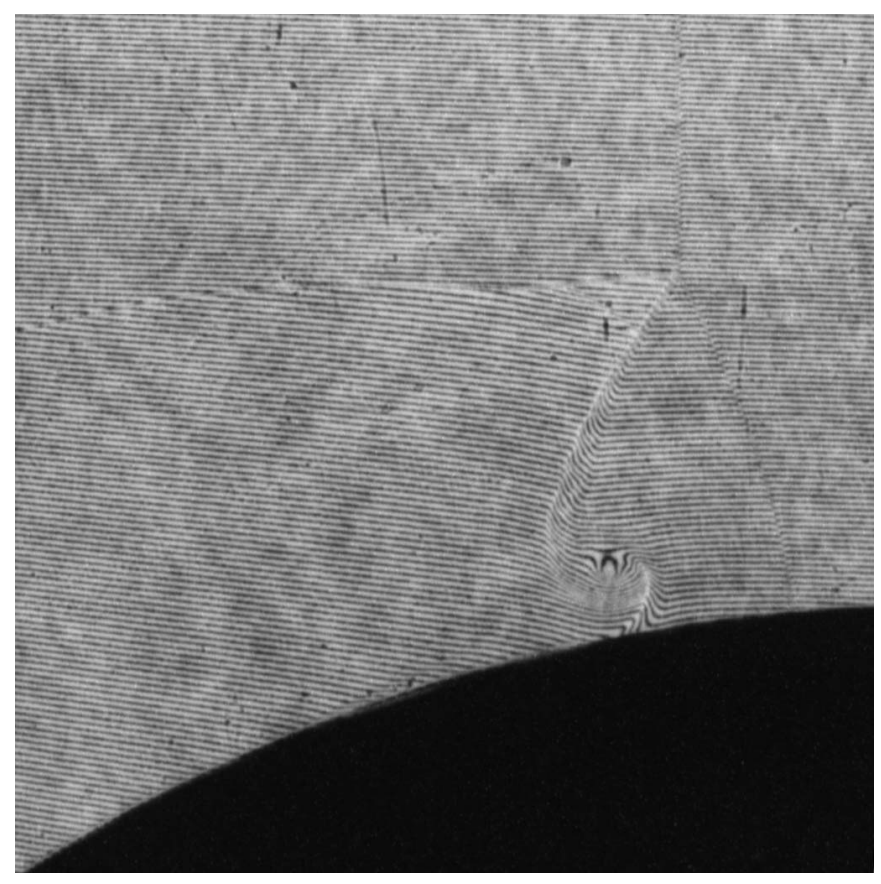

Fig. 14. Finite fringe interferogram of shock wave reflection off upper surface of semicircular model. The size of the imaged region is $94 \mathrm{~mm}$ in width $\times 134 \mathrm{~mm}$ in height.

distribution is represented in Fig. 15, whereas its magnitude is presented in Fig. 16.

Note that Fig. 15 has the appearance of an infinite fringe interferogram. The major difference is that noise, which would normally be present in such an interferogram, has been removed by the filtering in the Fourier transform plane. Figure 16 has the appearance of a shadowgraph that indicates that the observed shock configuration is a DMR, with the same features as those in Fig. 4.

The density distribution of the flowfield was determined by the application of Steps 5-6 and the use of Eq. (4). The result is presented in Fig. 17. In this figure, we identify a cut at $y=117.5 \mathrm{~mm}$ that we use for comparison with the CFD simulation for case b. This comparison is shown in Fig. 19 and discussed below. The origin of the Cartesian $(x, y)$ coordinate system used in Fig. 17 is on the stagnation point of the cylinder. The cut passes above $\mathrm{R}^{\prime}$ and below $\mathrm{T}$. In addition, it passes through $\mathrm{m}, \mathrm{s}, \mathrm{r}^{\prime}$ and $\mathrm{m}^{\prime}$.

\section{Uncertainty in normalized density}

We define $\eta=\frac{\rho_{\text {flow }}}{\rho_{\text {ref }}}$ to be the normalized measured density presented in Fig. 17.

The uncertainty $\delta \eta$ in the measured value of $\eta$ is dominated by the uncertainty in measuring the phase.

Hence, $\delta \eta$ is found from Eq. (4), to be:

$$
\delta \eta=\frac{\lambda p_{\mathrm{s}} T_{\mathrm{ref}}}{W p_{\mathrm{ref}} T_{\mathrm{s}} \beta} \frac{\delta \phi}{2 \pi}
$$




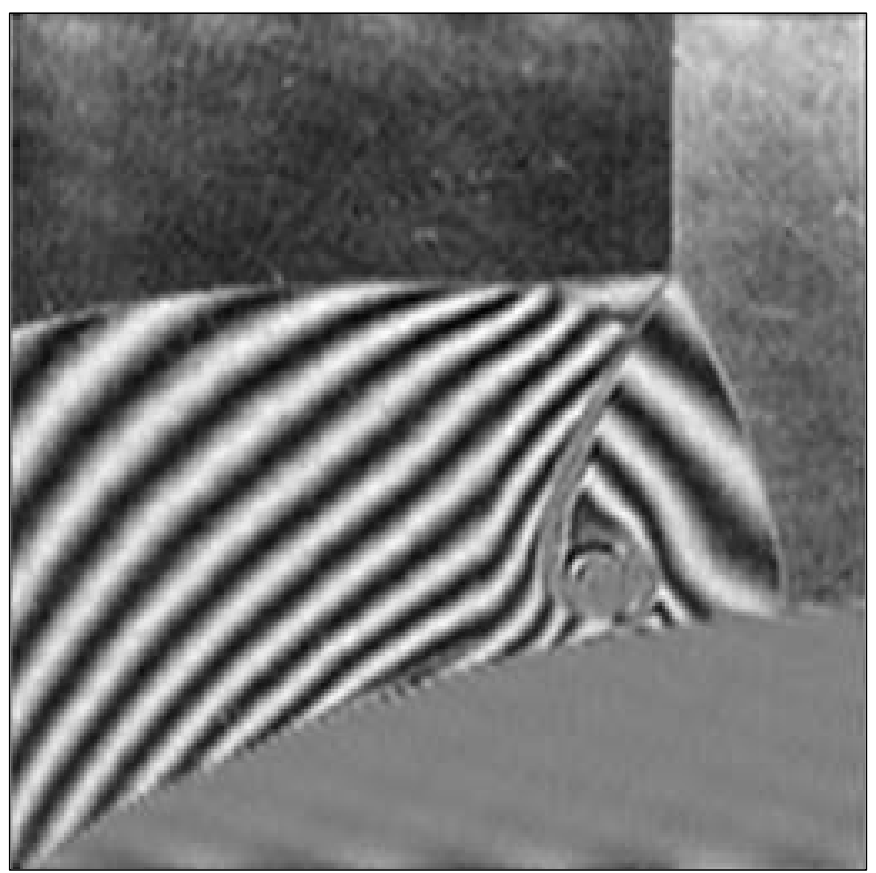

Fig. 15. Real part of $g$.

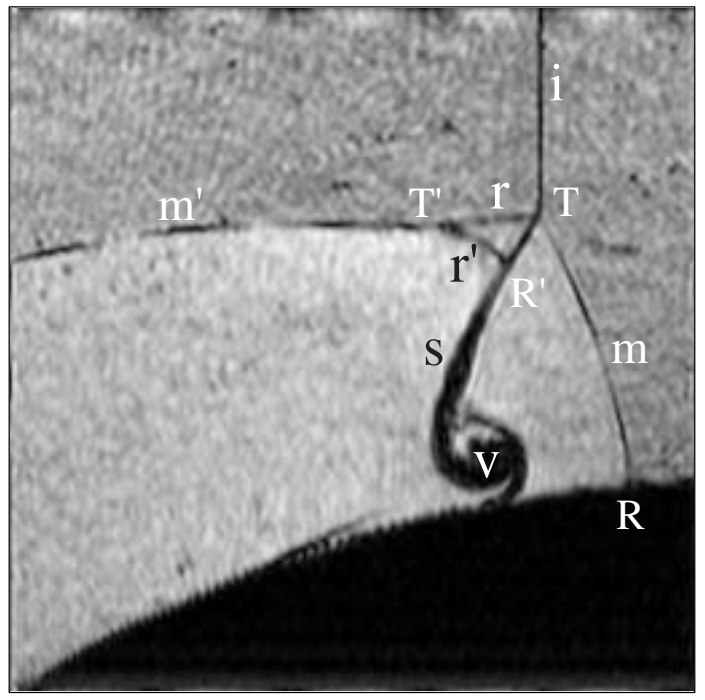

Fig. 16. Magnitude of $g$ : (letters indicate features identified in Fig. 4

where we have used the ideal equation of state to write density in terms of pressure and temperature and $\frac{\delta \phi}{2 \pi}$ is the uncertainty in the fringe shift.

The values used for the parameters in Eq. (5) to determine $\delta \eta$ in the current work are presented in Table 3

Bone et al. [10], on whose technique our fringe analysis is based, claim that for their method $\frac{\delta \phi}{2 \pi} \approx 0.02$ Using the values given in Table 3, we determine from Eq. (5) that the uncertainty $\delta \eta$ is less than $0.4 \%$

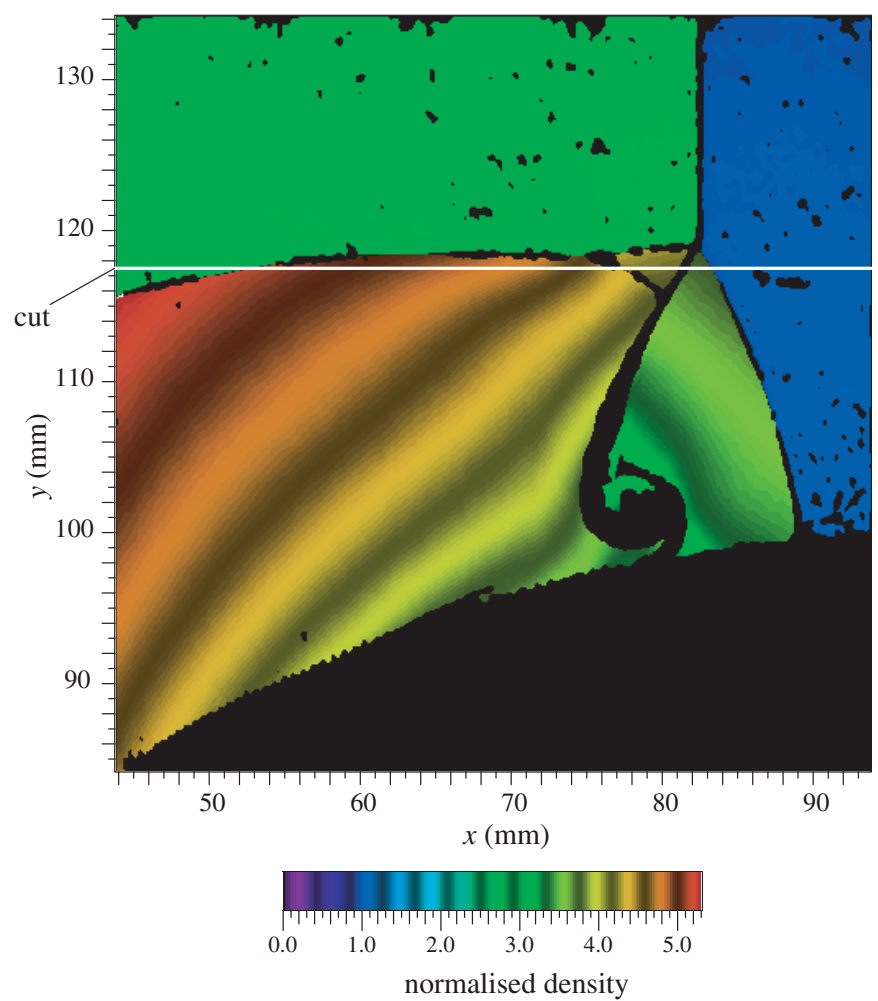

Fig. 17. Density Map of flowfield and cut at $y=117.5 \mathrm{~mm}$.

Table 3. Values used for the parameters in Eq. (5)

\begin{tabular}{llll}
\hline parameter & value & parameter & value \\
\hline$\lambda$ & $694.3 \mathrm{~nm}$ & $T_{\mathrm{s}}$ & $273 \mathrm{~K}$ \\
$W$ & $100 \mathrm{~mm}$ & $T_{\text {ref }}$ & $292 \mathrm{~K}$ \\
$p_{\mathrm{s}}$ & $101.13 \mathrm{kPa}$ & $\beta$ & $2.97 \times 10^{-4}$ \\
$p_{\text {ref }}$ & $14.1 \mathrm{kPa}$ & $\frac{\delta \phi}{2 \pi}$ & 0.02 \\
\hline
\end{tabular}

Table 4. Experimentally-determined locations of triple points, reflection points and vortex

\begin{tabular}{lllll}
\hline feature & $x(\mathrm{~mm})$ & $y(\mathrm{~mm})$ & $\Delta x(\mathrm{~mm})$ & $\Delta y(\mathrm{~mm})$ \\
\hline $\mathrm{R}$ & 89 & 100 & \pm 2 & \pm 2 \\
$\mathrm{R}^{\prime}$ & 80 & 116 & \pm 1 & \pm 1 \\
$\mathrm{~T}$ & 82.0 & 119.0 & \pm 0.5 & \pm 0.5 \\
$\mathrm{~T}^{\prime}$ & 75 & 119 & \pm 2 & \pm 2 \\
$\mathrm{v}$ & 79 & 101 & \pm 3 & \pm 3 \\
\hline
\end{tabular}

\subsection{Comparison of CFD simulation with experimental results}

Figure 18 shows the density contours of the flowfield as determined by the CFD simulation for case b. This simulation was produced using the method described above. The CFD simulation was initiated with the incident shock wave $20 \mathrm{~mm}$ upstream of the cylinder (i.e. with $\mathrm{i}$ at $y=-20 \mathrm{~mm}$ ) and terminated when the location of $i$ in the CFD coincided with its location in the experiment, that is, when $\frac{\Delta}{R}=0.825$. Note, that, in Fig. 18, the letters identify the same flow features as identified in Fig. 3 and that the simulation produces a TMR in contradiction to 
Table 5. Experimentally-determined locations of flow features along cut at $y=117.5 \mathrm{~mm}$. NA indicates that cut does not pass through feature. Each value has an uncertainty of $\pm 0.5 \mathrm{~mm}$

\begin{tabular}{lllll}
\hline$x_{m}(\mathrm{~mm})$ & $x_{m^{\prime}}(\mathrm{mm})$ & $x_{s}(\mathrm{~mm})$ & $x_{r}(\mathrm{~mm})$ & $x_{r^{\prime}}(\mathrm{mm})$ \\
\hline 83.1 & 56.5 & 81.4 & NA & 77.4 \\
\hline
\end{tabular}

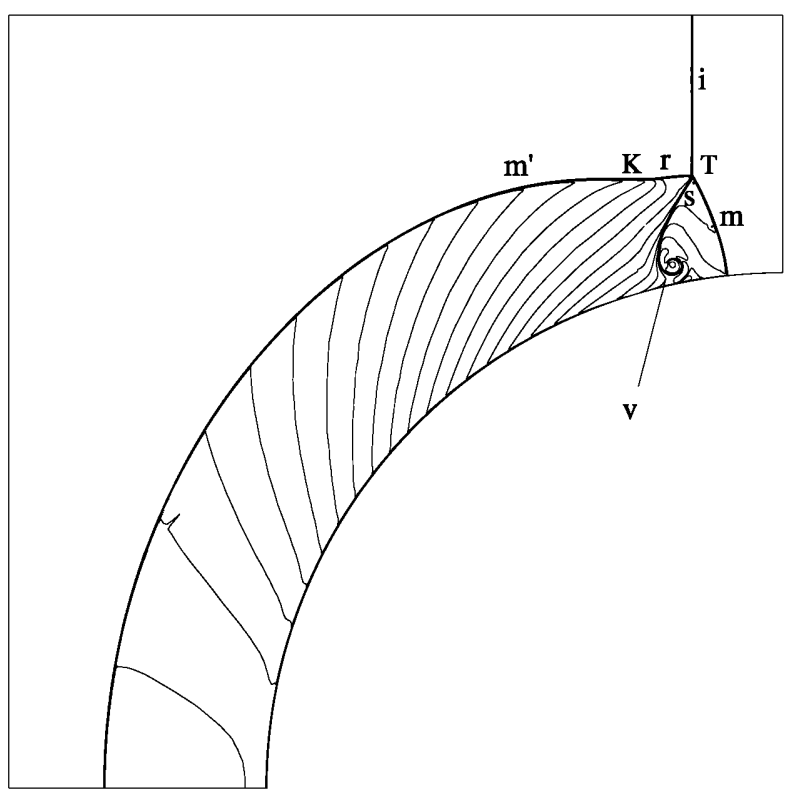

Fig. 18. Density contours of flowfield produced by CFD simulation for case b: letters identify the same flow features as in Fig. 3

the DMR observed in the experiment. This discrepancy is even more pronounced when we compare the density from the CFD simulation to that from the experimental measurements along the cut at $y=117.5 \mathrm{~mm}$, as described below.

In Fig. 19, we compare the experimentally-measured and CFD-generated density along the cut shown in Fig. 17. In Fig. 19, we see that the CFD follows the experimentally-observed density profile in a qualitative sense, in as much as, from right to left, both CFD and experiment show density increases across $\mathrm{m}$ and $\mathrm{s}$ and a density decrease across the rearward-facing shock $\mathrm{m}^{\prime}$. However, where the experiment shows a steep density rise across $\mathrm{r}^{\prime}$ the CFD predicts only a small density gradient, suggesting that the CFD simulates a compression wave rather than a shock wave. Further, there is a discrepancy between CFD and experiment in the location of $\mathrm{m}^{\prime}$. These discrepancies are because the CFD predicts TMR, whereas DMR is observed in the experiment. This could indicate that, in the CFD simulation, the incident shock did not impact the cylinder at exactly the same time and at exactly the same location as in the experiment. In fact, as discussed by Ben-Dor et al [4], whether or not the condition for DMR is satisfied is sensitively dependent on where the shock strikes the cylinder. If the correct initial conditions are not used by the CFD, it is possible that TMR is simulated instead of DMR. In the current work, the sensitivity of the solution to the initial position of the

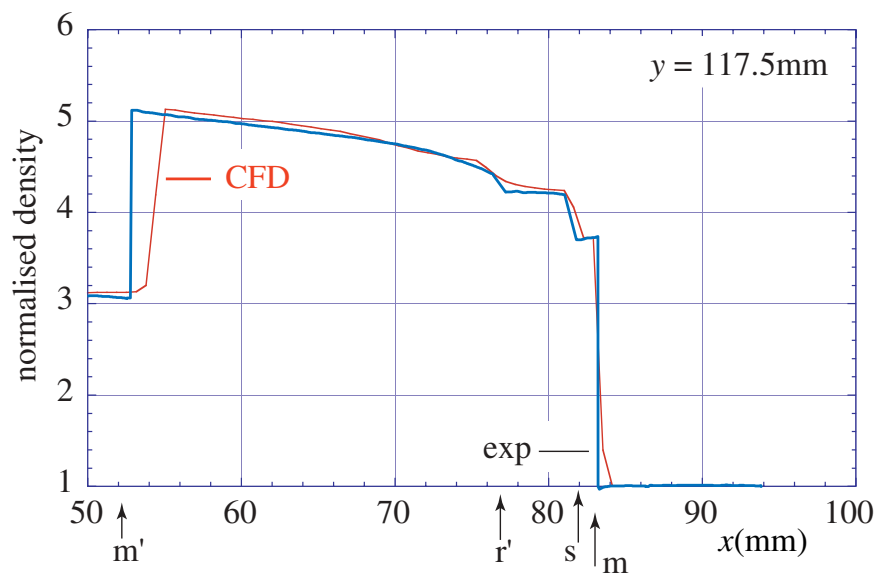

Fig. 19. Comparison between theoretical and experimental density for cut at $y=117.5 \mathrm{~mm}$. Letters indicate experimentally-measured locations of features identified in Fig. 16

incident shock was not explored, however, the discrepancy between CFD and experiment observed here indicates that this should be investigated in further numerical work. A possible cause for a difference in initial conditions is that the model might have moved soon after the incident shock struck its surface, causing the angle of incidence between surface and shock to be different in experiment from that in the CFD, thereby affecting the initial conditions of the shock reflection process in the experiment and possibly causing the experimental configuration to develop into one quite different from that predicted by CFD. This proposed explanation should be investigated in further work.

Three-dimensional effects can play a role in causing the discrepancy, if the gap between the semicircular cylinder and the window is sufficiently large, because such a gap could allow high pressure gas from below the model to cause compression waves to travel through the gap to the upper surface where they can strengthen the compression wave in a TMR configuration causing it to become a DMR configuration. However, this is unlikely to be the case in our cylinder model. The gap between the model and the window is the order of $0.1 \mathrm{~mm}$ The boundary layer on the side walls is another possible three-dimensional factor. However, it is very thin in the shock tube experiment.

\section{Conclusions}

This report has demonstrated the use of finite fringe holographic interferometry for the quantitative measurements of density in nonstationary shock wave reflection. The experimental results demonstrate our ability to resolve detailed structure in this complex shock wave reflection problem, allowing visualization of multiple shocks in the vicinity of the triple point, visualization of the shear layer and an associated vortical structure. The experimental results have been compared with a CFD simulation and discrepancies have been observed with CFD predicting transitional Mach reflection and experiment indicating double Mach reflection has developed. The inability of the CFD 
to reproduce the experimental observation has not been explained and is left as a possible topic for future research.

Acknowledgements. We thank O. Onodera, T. Ogawa and H. Ojima for their technical and professional expertise in assisting with the performance of the experiments described in this paper. A.F.P. Houwing gratefully acknowledges the financial support given to him as Visiting Professor at the Shock Wave Research Center at The Institute of Fluid Science at Tohoku University in Sendai to participate in the collaborative research project described in this paper. Furthermore, we express our appreciation to Drs. Holger Babinsky and Don Bone for making available the source listings of their numerical algorithms.

\section{References}

1. Caulfield, H.J.: Handbook of optical holography. Academic Press, ISBN 0121653501, 475 (1979)

2. Takayama, K.: Application of holographic interferometry to shock wave research. Proc. of the Society of PhotoOptical Instrumentation Engineers 398, 174-180 (1983)

3. Mitobe, H.: Experimental study of the transition from regular reflection to Mach reflection. MSc Thesis, Tohoku University(1999)

4. Ben-Dor, G., Igra, O., Elperin, T. (eds.): Handbook of Shock Waves 2, 163 (2001a)
5. Ben-Dor, G., Igra, O., Elperin, T. (eds.): Handbook of Shock Waves 2, 122 (2001b)

6. Ben-Dor, G., Igra, O., Elperin, T. (eds.): Handbook of Shock Waves 2, 126 (2001c)

7. Sun, M., Takayama, K.: Conservative smoothing on an adaptive quadrilateral grid. J. Comput. Phys. 150, 143180 (1999)

8. Sun, M., Takayama, K.: An artificially upstream flux vector splitting scheme for the Euler equations. J. Comput. Phys. 189, 305 (2003)

9. Sun, M., Yada, K., Jagadeesh, G., Onodera, O., Ogawa, T., Takayama, K.: A study of shock wave interaction with a rotating cylinder. Shock Waves Journal 12, 479-485 (2003)

10. Bone, D.J., Bachor, H.A., Sandeman, R.J.: Fringe-pattern analysis using a 2-D Fourier transform. Applied Optics 25 (10), 1653-1660 (1986)

11. Bone, D.J.: Fourier fringe analysis-the two-dimensional phase unwrapping problem. Applied Optics 30 (25), 36273632 (1991)

12. Babinsky, H., Takayama, K.: Quantitative holographic interferometry of shock-wave flows using Fourier transform fringe analysis. In: Proc. 20th Intn Sym. Shock Waves, Pasadena Jul, World Scientific Press, 1599-1604 (1995)

13. Merzkirch, W.:Flow visualisation., Academic Press, ISBN 0-12-491350-4 (1974)

14. Liepmann, H.W., Roshko, A.: Elements of Gasdynamics. John Wiley and Sons, New York, 154 (1957) 